\title{
The effect of surface topography on the micellisation of hexadecyltrimethylammonium chloride at the silicon-aqueous interface
}

\author{
R. Darkins ${ }^{1}$, M.L. Sushko², J. Liu ${ }^{2}$, D.M. Duffy ${ }^{1}$ \\ ${ }^{1}$ Department of Physics and Astronomy, University College London, Gower Street, \\ London, WC1E 6BT, UK \\ ${ }^{2}$ Pacific Northwest National Laboratory, Richland, Washington 99352, USA \\ E-mail: d.duffy@ucl.ac.uk
}

\begin{abstract}
Amphiphilic aggregation at solid-liquid interfaces can generate mesostructured micelles that can serve as soft templates. In this study we have simulated the self-assembly of hexadecyltrimethylammonium chloride $\left(\mathrm{C}_{16} \mathrm{TAC}\right)$ surfactants at the $\mathrm{Si}(100)$ - and $\mathrm{Si}(111)$-aqueous interfaces. The surfactants are found to form semicylindrical micelles on $\mathrm{Si}(100)$ but hemispherical micelles on $\mathrm{Si}(111)$. This difference in micelle structure is shown to be a consequence of the starkly different surface topographies that result from the reconstruction of the two silicon surfaces, and reveals that micelle structure can be governed by epitaxial matching even with non-polar substrates.
\end{abstract}

Keywords: self-assembly, ctac, silicon, micelle, topography 


\section{Introduction}

The aggregation of surfactants at solid-liquid interfaces can serve two general functions. One is to modify the interfacial forces, as in various industrial processes [1] such as detergency and ore flotation. The other is to generate mesostructured supramolecular assemblies such as micelles which can then act as soft templates. For example, mesoporous organosilicas are typically synthesised from an aqueous solution of structuredirecting surfactants and a silica precursor. These highly porous silica based materials are used in catalysis, sensing, and drug delivery [2] and they have been grown on substrates such as graphite [3], mica [4], and glass [5]. The surfactants are believed to self-assemble upon the substrate, forming a mesostructured template that subsequently aids nucleation and controls the orientation of the crystal.

The structure adopted by surfactant aggregates is influenced by many factors such as surfactant geometry, counterion, concentration, temperature, and $\mathrm{pH}[6,7]$. In this work we are concerned with the influence of the substrate. It is already wellestablished that different substrates give rise to different micelle structures. For example, under similar conditions, quaternary ammonium surfactants form semicylindrical micelles on graphite, hemispherical micelles on amorphous silica, and full cylinders on mica [8]. Different faces of the same crystal can also give rise to different micelle structures. For example, computer simulations of sodium dodecyl sulphate micellisation at the $\mathrm{TiO}_{2}$ (rutile)-water interface produced semicylindrical micelles on the (100) face, hemispherical micelles on (001), and rudimentary bilayers on (110) [9]. In a previous paper [10] we showed these assemblies to be directed predominantly by surface-surfactant electrostatic complementarity. Substrates can also control the orientation of the micelles through epitaxial registry. A well-known example of this

is the aforementioned semicylindrical micelles on graphite $[11,12,13]$ which align perpendicularly to the graphite symmetry axes.

In the present work we have simulated the micellisation of hexadecyltrimethylammonium chloride $\left(\mathrm{C}_{16} \mathrm{TAC}\right)$ surfactants at the silicon-aqueous interface for the nonoxidised, reconstructed $\mathrm{Si}(100)$ and $\mathrm{Si}(111)$ surfaces. The drivers for adsorption on these surfaces will differ significantly from that on many of the above examples. For instance, while adsorption on metal oxides are governed predominantly by ionic bonding, hydrogen bonding, and hydrophobic interactions [14], adsorption on silicon will be directed by soft epitaxy and induced image charges. Modelling image charges [15], however, is very challenging and we expect it to play a minor role in our system due to the predominance of the neutral alkane group in the surfactants and the mostly localised covalent bonding in silicon.

The main finding of our work is that the surfactants self-assemble to form semicylindrical micelles on $\mathrm{Si}(100)$ but hemispherical micelles on $\mathrm{Si}(111)$. While it has already been reported that organic molecules can achieve epitaxy with metal substrates [16], this work, to the best of our knowledge, presents the first reported instance of micelle morphology being determined by the topography of a non-polar substrate. We 
also note that semicylindrical micelles form domains [17] and herringbone patterns [18] on graphite due to its three symmetry axes, whereas $\mathrm{Si}(100)$ has only a single symmetry axis. This makes it a potentially attractive substrate for various applications such as nanopatterning and mesoporous materials synthesis.

\section{Methodology}

Four molecular dynamics simulations were performed in total. The first two consisted of $\mathrm{C}_{16}$ TAC surfactants at the $\mathrm{Si}(100)$-water and $\mathrm{Si}(111)$-water interfaces. They were then repeated but with the addition of sodium ions and silicic acid (detailed below).

In the first two simulations, a layer of $\mathrm{C}_{16} \mathrm{TA}^{+}$surfactants were positioned above the silicon surface and aligned normal to it in all-trans configuration with the hydrophilic headgroups pointing towards the surface. The chloride counterions were placed between the surface and the headgroups. The structure was then surrounded by a lattice of water molecules, with any overlapping molecules removed. The amount of water was chosen such that the thickness of the water layer, upon relaxation, was approximately $5 \mathrm{~nm}$. A vacuum slab of $5 \mathrm{~nm}$ was then added above the water; in addition to segregating the periodic images, the vacuum slab enabled the system to maintain zero pressure while sampling the canonical ensemble.

The concentration of surfactants at an interface is known to vary non-linearly with the bulk concentration, as described by the adsorption isotherm. We aimed to reproduce a bulk concentration of $2.0 \mathrm{mM} \mathrm{C}_{16} \mathrm{TAC}$ which is slightly above the experimentallydetermined critical micelle concentration of $1.3 \mathrm{mM}$ [19]. However, unaware of any published $\mathrm{C}_{16} \mathrm{TAC} /$ silicon isotherms, we utilised $\mathrm{C}_{16} \mathrm{TAC} /$ silica isotherms [20] as a reasonable estimate which gave an adsorption density of $1.9 \mathrm{~nm}^{-2}$ at the desired bulk concentration of $2.0 \mathrm{mM} \mathrm{C}_{16} \mathrm{TAC}$.

In an experimental context, surfactants are invariably added to a solution in the presence of other molecules. We consider mesoporous organosilica as a representative system, the synthesis of which typically involves reacting a silica source, such as sodium silicate, with a structure-directing alkytrimethylammonium halide surfactant under basic conditions [21, 22, 23]. To replicate this, sodium ions and silicic acid monomers were added to the water in our second batch of simulations with respective $\mathrm{Na}^{+}: \mathrm{SiO}_{4} \mathrm{H}_{3}^{-}: \mathrm{C}_{16}$ TAC concentrations of $1: 1: 1$. The silicic acid was assumed to be deprotonated $\left(\mathrm{SiO}_{4} \mathrm{H}_{3}^{-}\right)$which is mostly true under basic conditions, and we disregard the presence of silica oligomers since the process of oligomerisation occurs on a much longer time-scale than we are simulating [24]. These impurities were mixed with the chloride counterions and scattered in the volume above the $\mathrm{C}_{16} \mathrm{TA}^{+}$surfactants. In an attempt to minimise the net dipole moment inevitably built into our initial configuration, the surfactants were inverted so that the headgroups pointed away from the surface.

All four systems were then simulated at $300 \mathrm{~K}$ for $5 \mathrm{~ns}$ each. Further details are provided in the following three sections. 
(a)
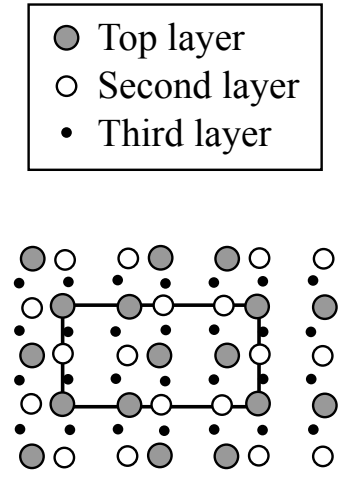

(b)

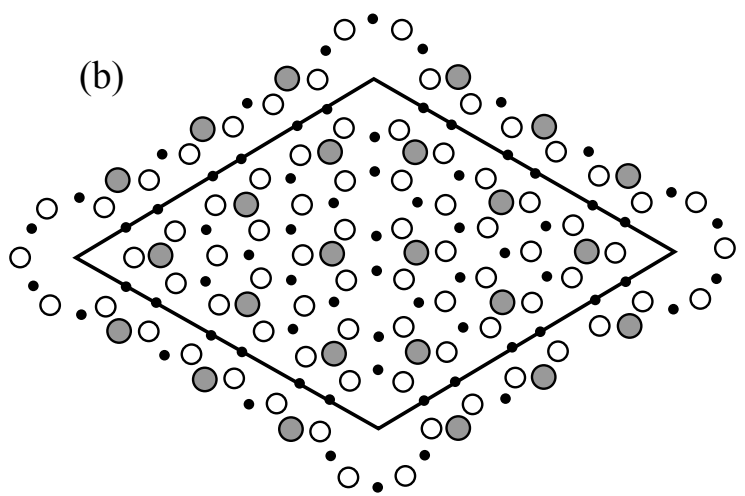

Figure 1. Structure of the reconstructed (a) $\operatorname{Si}(100)-c(4 \times 2)$ and (b) $\operatorname{Si}(111)-p(7 \times 7)$ surfaces with the conventional unit cells outlined.

\subsection{Silicon surfaces}

Both $\mathrm{Si}(100)$ and $\mathrm{Si}(111)$ are known to undergo significant reconstruction, as depicted in Figure 1. In the case of $\mathrm{Si}(100)$ we adopted the $\mathrm{c}(4 \times 2)$ buckled configuration which was created using density functional theory (DFT), details of which can be found in the next section. We note that while there remains some debate regarding whether or not the surface dimers buckle experimentally [25, p. 268], the buckling appears to be irrelevant to our findings. In the case of $\mathrm{Si}(111)$, the surface undergoes a very elaborate reconstruction that requires a large $\mathrm{p}(7 \times 7)$ unit cell, making it impractical for us to build this surface with DFT as we did for $\mathrm{Si}(100)$. Instead, the configuration was constructed by reproducing the geometry determined from low-energy electron diffraction analysis $[26]$.

In our simulations, surfaces larger than a single unit cell were used. For $\mathrm{Si}(100)$, the slab was composed of $4 \times 8$ unit cells which had dimensions of approximately $6.2 \times 6.2$ $\mathrm{nm}$. The $\mathrm{Si}(111)$ slab consisted of $2 \times 2$ unit cells and had cell vectors of length $8.1 \mathrm{~nm}$ and at an angle of $54.7^{\circ}$ to each other. These slabs were specifically chosen to be large enough to support both semicylindrical and hemispherical micelles so as not to bias the final micelle structure.

\subsection{Interatomic potentials}

The $\mathrm{C}_{16} \mathrm{TA}^{+}$surfactants and the chloride and sodium ions were all described using the GROMOS96 G45a3 [27, 28] force field which is a united carbon atom model, meaning that any hydrogen atoms are fused with their parent carbon atom to form a single interaction site. These potentials have been employed in several recent studies of alkytrimethylammonium halide micellisation [29, 30, 31, 32, 33, 34]. The silicic acid was modelled with the potentials of Jorge et al. [31] and the water with the rigid SPC/E model [35]. The silicon surfaces were fixed in the simulations and so no Si-Si interactions 


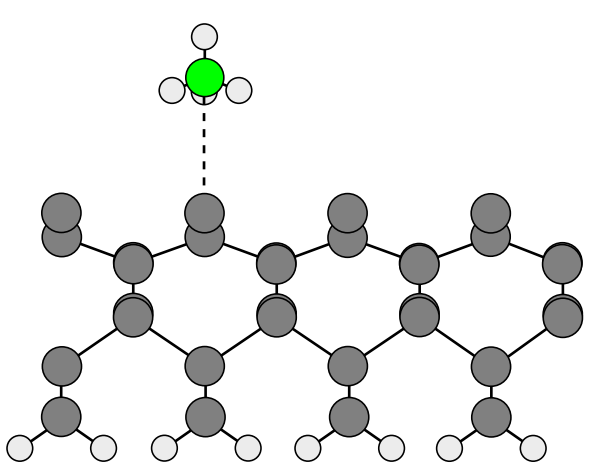

(a)

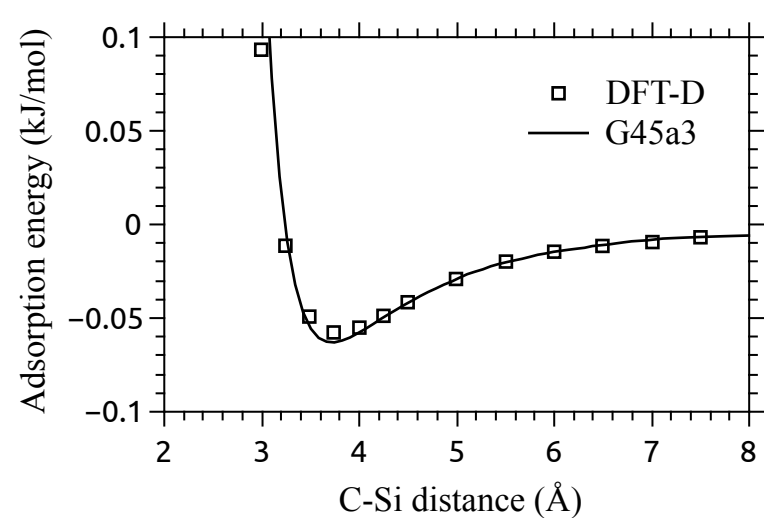

(b)

Figure 2. (a) A schematic representation of the atomic model used to validate the silicon potentials. A methane molecule is positioned above a silicon atom on the top layer of $\mathrm{Si}(100)$ and the energy-distance curve is computed. (b) The resulting adsorption curve for the DFT-D (squares) and the classical G45a3 (line) calculations.

were required.

The interactions between the surface $\mathrm{Si}$ atoms and all other atoms were modelled using the Lennard-Jones potential parameters also provided by G45a3. In order to validate that these potentials were appropriate for the current study we compared the adsorption energy curves for a $\mathrm{CH}_{4}$ molecule on a Si surface calculated using the classical potentials with those computed using DFT.

The DFT calculations were performed using a five layer slab of $\mathrm{Si}(100)$ composed of $2 \mathrm{x} 1$ lateral unit cells, with dimensions of $15.47 \times 15.47 \AA$, constructed using the procedure in Ref [36]. Each silicon atom in the bottom layer was passivated with a pair of hydrogen atoms. The slab was periodic in three dimensions with a vacuum slab of $20 \AA$ added to attenuate self-interaction between the images in the $z$-dimension. A methane $\left(\mathrm{CH}_{4}\right)$ molecule was then positioned directly atop an uppermost silicon atom and the carbon-silicon distance varied, as illustrated in Figure 2(a). The difference in energy with respect to an isolated surface and methane molecule was then recorded. The calculations were performed using DFT as implemented in Quantum Espresso 4.1 [37]. The generalised gradient approximation based Perdew, Burke, and Ernzerhof (PBE) exchange-correlation functional was employed. The core electrons were modelled with Troullier-Martins [38] norm-conserving pseudopotentials with the configurations $\left[1 \mathrm{~s}^{2}\right] 2 \mathrm{~s}^{2}$ $2 \mathrm{p}^{2}$ and $[\mathrm{Ne}] 3 \mathrm{~s}^{2} 3 \mathrm{p}^{2}$ for carbon and silicon, respectively. Only the $\Gamma$-point was sampled and a kinetic energy cutoff of 30 Ry was used. Due to the locality of the exchangecorrelation functional, intermolecular dispersion effects are not directly reproduced. To account for this we used the DFT-D dispersion correction of Grimme et al. [39] which consists of a parametrised pairwise $r^{-6}$ energy contribution. The computed adsorption energies are plotted in Figure 2(b).

The adsorption energy was computed in an analogous way atomistically for the same silicon surface (minus the hydrogen atoms) and for the G45a3 model of the $\mathrm{CH}_{2}$ 

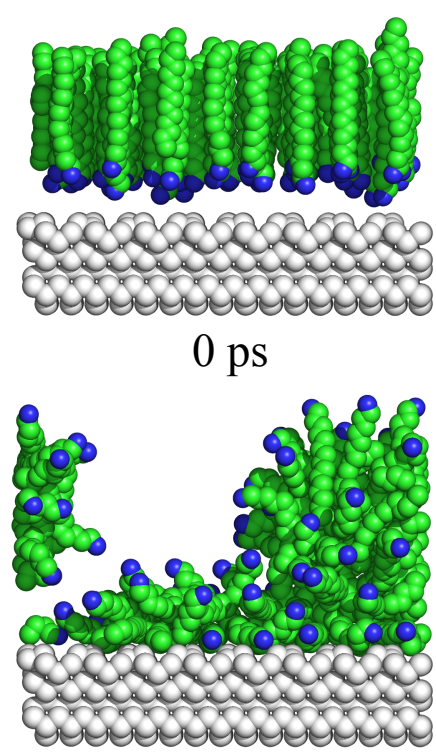

$300 \mathrm{ps}$

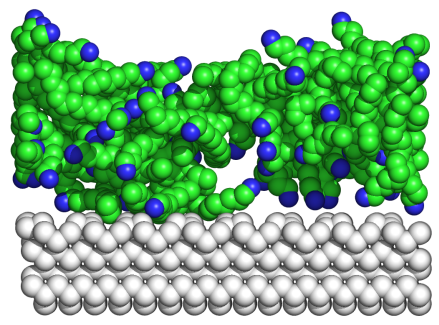

100 ps

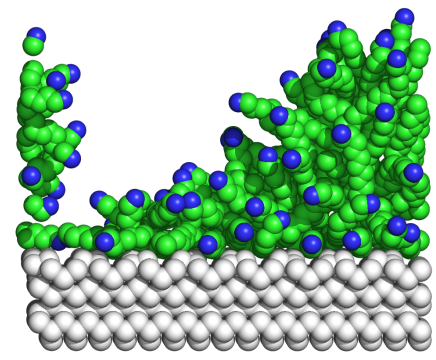

400 ps
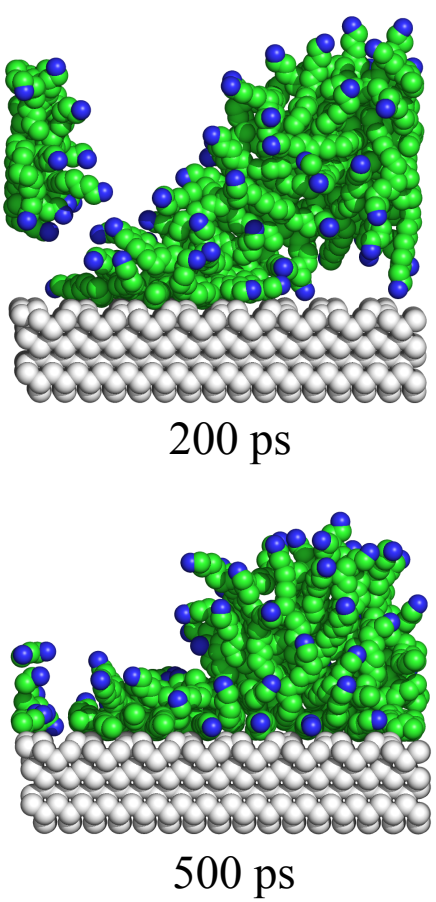

Figure 3. Time-evolution of the surfactants at the $\operatorname{Si}(100) /$ water interface. A rudimentary semicylindrical micelle is formed. The silicon surface is shown in grey, the alkane tail in green, and the nitrogen atom from the headgroup in blue. All other atoms are hidden for clarity.

molecule which is a constituent of the alkane tails. The resulting energy curve is also plotted in Figure 2(b). There is very good agreement between the two sets of data. Both give the same energy minimum and equilibrium spacing to within $0.01 \mathrm{~kJ} / \mathrm{mol}$ and $0.2 \AA$, respectively. We conclude that the G45a3 silicon parameters are suitable for modelling silicon surfaces.

\subsection{Molecular dynamics details}

The simulations were performed using DL_POLY Classic 1.9 [40] in the NVT ensemble. Rhombohedral periodic boundary conditions were imposed and long-range electrostatics were handled by the particle mesh Ewald method. The Nosé-Hoover thermostat maintained the desired temperature. The leapfrog Verlet algorithm with a time-step of 2 fs was used to integrate the equations of motion.

\section{Results and discussion}

In the initial configurations of the pure-water simulations, the hydrophobic tails were exposed to the water above. Within the first 200 ps many of the surfactants had inverted and aggregated in an attempt to shield their tails from the water. In the case of $\mathrm{Si}(100)$, this resulted in an elongated micelle that was partially attached to the surface and which increased its contact over the subsequent 300 ps, as shown in Figure 3. This 

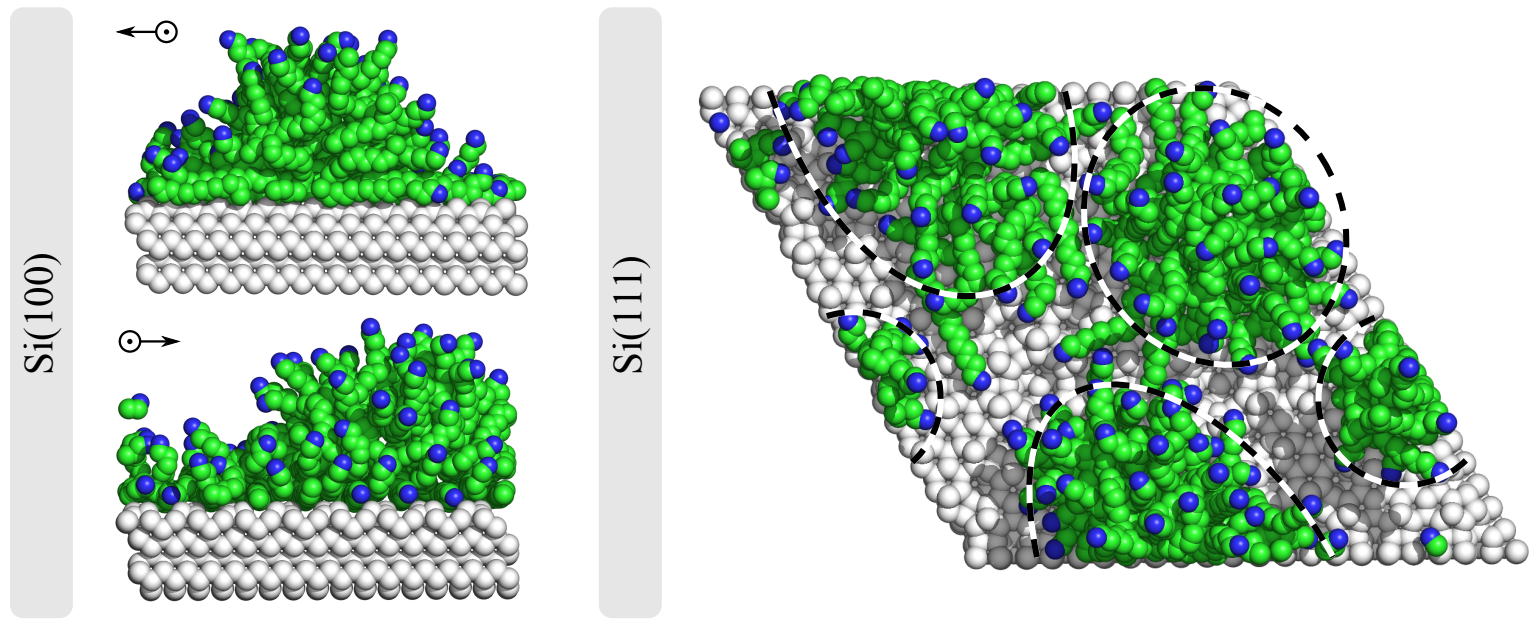

Figure 4. Side-views of the semicylindrical micelle formed on $\mathrm{Si}(100)$, and an overhead view of the hemispherical micelles (highlighted for clarity) formed on $\operatorname{Si}(111)$. These are the final configurations after 5 ns of simulation. See Figure 3 for the colour scheme.

process of aggregation, followed by a gradually increasing surface contact, also unfolded at the $\mathrm{Si}(111)$ interface except that, due to the larger unit cell and therefore the larger number of surfactants, three separate micelles were created (composed of 13, 35, and 58 surfactants).

The aggregation of the surfactants and their subsequent adsorption to the surfaces would have been driven partly, if not mostly, by entropy. This is because both processes are accompanied by the release of confined water molecules. Such a discharge increases the degrees of freedom of the system and therefore the entropy. In fact, in bulk $\mathrm{C}_{16} \mathrm{TAC}$ micellisation, over $65 \%$ of the free energy change is due to an increase in solvation entropy [19].

A crucial difference between the micellisation at the $\mathrm{Si}(100)$ and $\mathrm{Si}(111)$ interfaces was the organisation of the surfactants as they came into contact with the surfaces. On $\mathrm{Si}(100)$ the surfactants successively aligned in parallel, driven partly by their interaction with the preceding surfactants, resulting in a linear array that naturally gave rise to a semicylindrical structure. This final structure, shown in Figure 4, is slightly incomplete
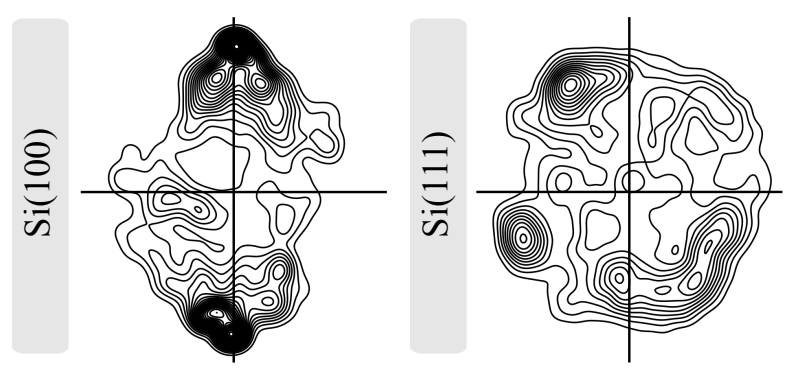

Figure 5. Density in the $x y$-plane of the vector between the headgroup and tail of each surfactant on the two substrates, $\mathrm{Si}(100)$ and $\mathrm{Si}(111)$. The respective densities are characteristic of semicylindrical and hemispherical micelles. 

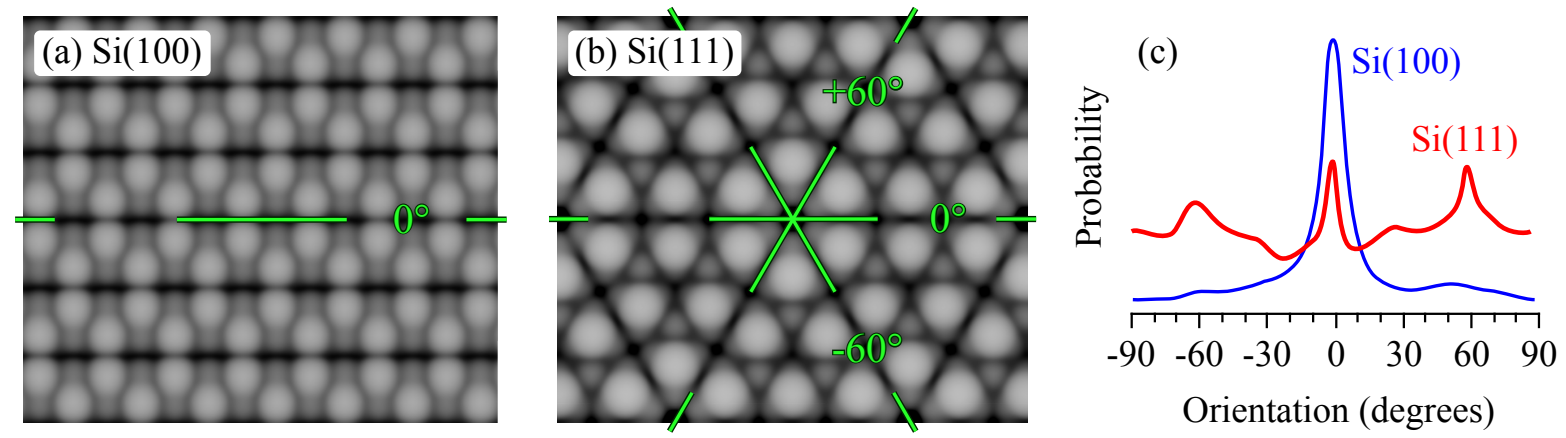

Figure 6. The topography of (a) $\operatorname{Si}(100)$ and (b) $\mathrm{Si}(111)$ from the perspective of a $-\mathrm{CH}_{2}$ - unit in the surfactant tail. The long surface crevices are highlighted in green.

(c) The angle distribution of the surfactants that fall within $1 \mathrm{~nm}$ of each surface.

suggesting that our (tentative) choice of surfactant concentration was indeed too low.

By contrast, the surfactants on $\mathrm{Si}(111)$ exhibited no such linear organisation and instead dispersed radially outward as they increased their coverage of the surface. This resulted in a pair of hemispherical micelles (the smallest of the three micelles remained detached from the surface) as shown in Figure 4 . The densities in the $x y$-plane of the vector between the headgroup and tail of each surfactant on the two substrates, $\mathrm{Si}(100)$ and $\mathrm{Si}(111)$, are shown in Figure 5. The respective densities are characteristic of semicylindrical and hemispherical micelles.

Repeating these simulations but with the sodium and silanol added to the solution presented a practical hurdle. The initial configuration had a net dipole moment which the system subsequently quenched by pulling the $\mathrm{CTA}^{+}$molecules away from the surface and into the ionic mix above. In doing so, a micelle formed that was completely detached from the surface and which, due to its stability, would have taken prohibitively long to reattach. We therefore restarted the simulations but having equilibrated them first for 500 ps with the tail-ends of the $\mathrm{CTA}^{+}$molecules fixed near to the surfaces. This allowed the ions and the headgroups to reduce the dipole without forming a micelle in the process. When the tails were released, the surfactants relaxed as before, forming a semicylindrical micelle on $\mathrm{Si}(100)$ and hemispherical micelles on $\mathrm{Si}(111)$. Some of the chloride counterions in the vicinity of the $\mathrm{CTA}^{+}$headgroups were replaced with silicic acid while the remaining ions eventually aggregated to form ionically-bound oligomeric chains. These neutral aggregates remained detached from the micelles which would explain their impotence in affecting the resulting structures. The fact that this second batch of simulations resulted in the same morphologies as the first would suggest that the structures were not biased by the initial configurations.

\subsection{Role of surface topography}

To explain the different micelle structures that form on $\mathrm{Si}(100)$ and $\mathrm{Si}(111)$ we have analysed the topographies of the two surfaces. Figures 6(a) and (b) show the variation in adhesion energy between each silicon surface and a single $-\mathrm{CH}_{2}-$ molecule from the 
alkane tails, where dark regions correspond to strong adhesion. This was computed by sweeping the $-\mathrm{CH}_{2}$ - probe across a two-dimensional grid and then, for each $(x, y)$ coordinate, minimising the energy in the $z$ direction. We have also computed the orientation of the surfactants near to the surfaces. More specifically, for each unit $\mathbf{x}_{i}$ of the hydrocarbons within $1 \mathrm{~nm}$ of the surface, we projected $\mathbf{x}_{i+1}-\mathbf{x}_{i-1}$ onto the $x y$-plane and computed the angle with respect to the $+x$ axis. The time-averaged distribution of these angles is shown in Figure 6(c) for both surfaces.

$\mathrm{Si}(100)$ displays a distinct corrugation with rows of crevices. The surfactants evidently align themselves with these features and thus adopt a commensurate structure. A similar attempt is made by the surfactants on $\mathrm{Si}(111)$ to match the long crevices, as evidenced by the peaks at $-60^{\circ}, 0^{\circ}$, and $+60^{\circ}$ in Figure $6(\mathrm{c})$. By attempting to match the topography and symmetry of the surfaces, the hemispherical and semicylindrical structures naturally ensue.

When a semicylindrical micelle forms at the graphite-aqueous interface, it aligns with one of the three equivalent symmetry axes of the crystal plane. This alignment is believed to result from the fortuitous match between the distance of the centres of the hexagons $(2.46 \AA)$ and the distance between alternate units of the hydrocarbon chains $(2.51 \AA)$ giving rise to efficient chain packing and strong adhesion [41]. We have discovered a similar fortuity regarding hydrocarbons on $\mathrm{Si}(100)$, albeit inter- rather than intra-molecular in nature. Figure 7(a) shows the $z$-density of the hydrocarbons at the $\mathrm{Si}(100)$-water interface. The peaks reveal two distinct layers on the surface, vertically separated by $0.77 \AA$. By measuring the features of the $\mathrm{Si}(100)$ surface, we find that the hydrocarbons must be horizontally separated by an average of $3.86 \AA$. This arrangement is illustrated in Figure 7(b) and it follows that the average distance between the hydrocarbons in contact with the surface is $3.94 \AA$. This is less than 0.1 $\AA$ short of the mode separation of all of the hydrocarbons in the micelle, as shown in Figure $7(\mathrm{c})$. The hydrocarbons are therefore able to achieve registry with the substrate without straining.

The applicability of our findings to an experimental setting will, of course, depend on how the silicon surfaces are prepared. When silanol groups form on the surfaces they prevent direct interaction between the silicon and the surfactants. This can reduce the anisotropy and thus eliminate any influence from the surface topography on the micelle structure [42].

\section{Conclusions}

We have simulated the micellisation of hexadecyltrimethylammonium chloride surfactants at the $\mathrm{Si}(100)$ - and $\mathrm{Si}(111)$-aqueous interfaces both with and without sodium silicate ions. In all cases the surfactants aggregated and adsorbed to the surfaces forming distinct micelle structures with the hydrophobic groups shielded from the water. On $\mathrm{Si}(100)$ they adopted a semicylindrical structure but on $\mathrm{Si}(111)$ they were hemispherical. Comparing the structure and orientation of the adsorbed surfactants to the 


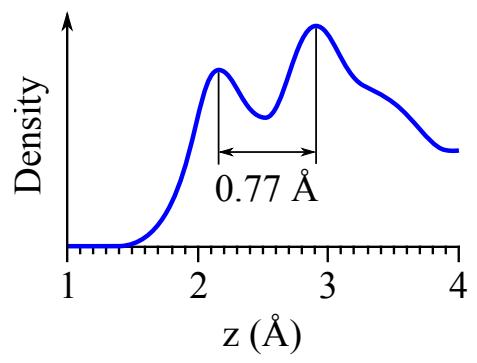

(a)

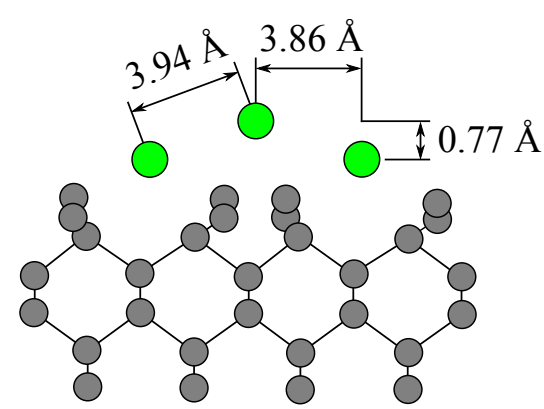

(b)

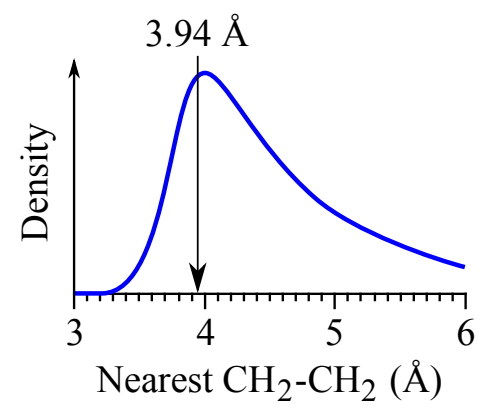

(c)

Figure 7. The structure of the surfactants adsorbed to $\mathrm{Si}(100)$. (a) Two distinct layers visible in the $z$-density of the surfactants. (b) The geometry of the surfactants (green, aligned normal to the page) with respect to the $\mathrm{Si}(100)$ surface. (c) A histogram showing the intermolecular distance between each $\mathrm{CH}_{2}$ and its nearest counterpart. The arrow indicates the average spacing between neighbouring surfactants adsorbed on $\mathrm{Si}(100)$, as shown in (b).

topographies of the surfaces revealed that the surfactants adopted their respective morphologies in an attempt to match the symmetry of the substrates. We also observed that, in the case of $\mathrm{Si}(100)$, the surfactants were fortuitously able to achieve perfect registry with the surface features without straining, suggesting a strong driving force for organisation.

Our results demonstrate that epitaxy can play a crucial role in determining micelle structure even in the case of non-polar surfaces with which the surfactants interact weakly via van der Waals forces.

Due to the existence of only a single symmetry axis for the reconstructed $\operatorname{Si}(100)$ surface, one would expect uniform self-assembly of surfactants at all length-scales making it potentially well-suited as a substrate for a range of applications particularly in the synthesis of mesoporous materials.

\section{Acknowledgements}

RD acknowledges funding from EPSRC under the Molecular Modelling and Materials Science Industrial Doctorate Centre for force-field testing and the US Department of Energy (DOE), Office of Basic Energy Sciences, Division of Materials Sciences and Engineering, under Award KC020105-FWP12152 for molecular simulation of surfactant self-assembly. MLS and JL acknowledge DOE support under the same award. PNNL is a multiprogram national laboratory operated for DOE by Battelle under Contract DE-AC05-76RL01830.

\section{References}

[1] David Robert Karsa. Industrial applications of surfactants IV. Number 230. Elsevier, 1999. 
[2] Frank Hoffmann, Maximilian Cornelius, Jürgen Morell, and Michael Fröba. Silica-Based Mesoporous Organic-Inorganic Hybrid Materials. Angewandte Chemie International Edition, 45(20):3216-3251, 2006.

[3] Hong Yang, Neil Coombs, Igor Sokolov, and Geoffrey A Ozin. Registered growth of mesoporous silica films on graphite. J. Mater. Chem., 7(7):1285-1290, 1997.

[4] Hong Yang, Alex Kuperman, Neil Coombs, Suzan Mamiche-Afara, and Geoffrey A Ozin. Synthesis of oriented films of mesoporous silica on mica. Nature, 379(6567):703-705, 1996.

[5] Hirokatsu Miyata and Kazuyuki Kuroda. Formation of a continuous mesoporous silica film with fully aligned mesochannels on a glass substrate. Chemistry of Materials, 12(1):49-54, 2000.

[6] Jacob N Israelachvili, D John Mitchell, and Barry W Ninham. Theory of self-assembly of hydrocarbon amphiphiles into micelles and bilayers. Journal of the Chemical Society, Faraday Transactions 2: Molecular and Chemical Physics, 72:1525-1568, 1976.

[7] Rui Zhang and P Somasundaran. Advances in adsorption of surfactants and their mixtures at solid/solution interfaces. Advances in colloid and interface science, 123:213-229, 2006.

[8] Srinivas Manne and Hermann E Gaub. Molecular organization of surfactants at solid-liquid interfaces. Science, 270(5241):1480-1482, 1995.

[9] E. Núñez-Rojas and H. Domínguez. Computational studies on the behavior of Sodium Dodecyl Sulfate (SDS) at TiO2(rutile)/water interfaces. Journal of colloid and interface science, 364(2):417-427, 2011.

[10] Robert Darkins, Maria L Sushko, Jun Liu, and Dorothy M Duffy. Adhesion of Sodium Dodecyl Sulfate Surfactant Monolayers with TiO2 (Rutile and Anatase) Surfaces. Langmuir, 29(37):11609-11614, 2013.

[11] S Manne, JP Cleveland, HE Gaub, GD Stucky, and PK Hansma. Direct visualization of surfactant hemimicelles by force microscopy of the electrical double layer. Langmuir, 10(12):4409-4413, 1994.

[12] Heather N Patrick, Gregory G Warr, Srinivas Manne, and Ilhan A Aksay. Self-assembly structures of nonionic surfactants at graphite/solution interfaces. Langmuir, 13(16):4349-4356, 1997.

[13] Rob Atkin and Gregory G Warr. Self-assembly of a nonionic surfactant at the graphite/ionic liquid interface. Journal of the American Chemical Society, 127(34):11940-11941, 2005.

[14] Siddharth V Patwardhan, Fateme S Emami, Rajiv J Berry, Sharon E Jones, Rajesh R Naik, Olivier Deschaume, Hendrik Heinz, and Carole C Perry. Chemistry of aqueous silica nanoparticle surfaces and the mechanism of selective peptide adsorption. Journal of the American Chemical Society, 134(14):6244-6256, 2012.

[15] Hendrik Heinz, Kshitij C Jha, Jutta Luettmer-Strathmann, Barry L Farmer, and Rajesh R Naik. Polarization at metal-biomolecular interfaces in solution. Journal of The Royal Society Interface, page rsif20100318, 2010.

[16] Jie Feng, Ras B Pandey, Rajiv J Berry, Barry L Farmer, Rajesh R Naik, and Hendrik Heinz. Adsorption mechanism of single amino acid and surfactant molecules to $\mathrm{Au}\{111\}$ surfaces in aqueous solution: design rules for metal-binding molecules. Soft Matter, 7(5):2113-2120, 2011.

[17] Sai-Long Xu, Chen Wang, Qing-Dao Zeng, Peng Wu, Zhi-Gang Wang, Hai-Ke Yan, and Chun-Li Bai. Self-assembly of cationic surfactants on a graphite surface studied by STM. Langmuir, 18(3):657-660, 2002.

[18] Vamsi K Paruchuri, Jakub Nalaskowski, Dinesh O Shah, and Jan D Miller. The effect of cosurfactants on sodium dodecyl sulfate micellar structures at a graphite surface. Colloids and Surfaces A: Physicochemical and Engineering Aspects, 272(3):157-163, 2006.

[19] Jitendra Mata, Dharmesh Varade, and Prashant Bahadur. Aggregation behavior of quaternary salt based cationic surfactants. Thermochimica Acta, 428(1):147-155, 2005.

[20] R Atkin, VSJ Craig, EJ Wanless, and S Biggs. The influence of chain length and electrolyte on the adsorption kinetics of cationic surfactants at the silica-aqueous solution interface. Journal of colloid and interface science, 266(2):236-244, 2003.

[21] VV Guliants, MA Carreon, and YS Lin. Ordered mesoporous and macroporous inorganic films 
and membranes. Journal of Membrane Science, 235(1):53-72, 2004.

[22] Chaojie Song and Gilles Villemure. Electrode modification with spin-coated films of mesoporous molecular sieve silicas. Microporous and mesoporous materials, 44:679-689, 2001.

[23] Shiyou Guan, Shinji Inagaki, Tetsu Ohsuna, and Osamu Terasaki. Cubic hybrid organic-inorganic mesoporous crystal with a decaoctahedral shape. Journal of the American Chemical Society, 122(23):5660-5661, 2000.

[24] Alon V McCormick, Alexis T Bell, and CJ Radke. Quantitative determination of siliceous species in sodium silicate solutions by ${ }^{29} \mathrm{Si}$ NMR spectroscopy. Zeolites, 7(3):183-190, 1987.

[25] Richard M Martin. Electronic structure: basic theory and practical methods. Cambridge university press, 2004.

[26] H Huang, SY Tong, WE Packard, and MB Webb. Atomic geometry of Si (111) $7 \times 7$ by dynamical low-energy electron diffraction. Physics Letters A, 130(3):166-170, 1988.

[27] Wilfred F van Gunsteren, SR Billeter, AA Eising, Philippe H Hünenberger, PKHC Krüger, Alan E Mark, WRP Scott, and Ilario G Tironi. Biomolecular simulation: The \{GROMOS96\} manual and user guide. 1996.

[28] Walter RP Scott, Philippe H Hünenberger, Ilario G Tironi, Alan E Mark, Salomon R Billeter, Jens Fennen, Andrew E Torda, Thomas Huber, Peter Krüger, and Wilfred F van Gunsteren. The GROMOS biomolecular simulation program package. The Journal of Physical Chemistry A, 103(19):3596-3607, 1999.

[29] Miguel Jorge. Molecular dynamics simulation of self-assembly of n-Decyltrimethylammonium Bromide micelles. Langmuir, 24(11):5714-5725, 2008.

[30] Germán Pérez-Sánchez, José RB Gomes, and Miguel Jorge. Modeling Self-Assembly of Silica/Surfactant Mesostructures in the Templated Synthesis of Nanoporous Solids. Langmuir, 29(7):2387-2396, 2013.

[31] Miguel Jorge, José RB Gomes, M Natália DS Cordeiro, and Nigel A Seaton. Molecular dynamics simulation of the early stages of the synthesis of periodic mesoporous silica. The Journal of Physical Chemistry B, 113(3):708-718, 2008.

[32] Miguel Jorge, José RB Gomes, M Natália DS Cordeiro, and Nigel A Seaton. Molecular simulation of silica/surfactant self-assembly in the synthesis of periodic mesoporous silicas. Journal of the American Chemical Society, 129(50):15414-15415, 2007.

[33] Ryusuke Futamura, Miguel Jorge, and José RB Gomes. Role of the organic linker in the early stages of the templated synthesis of PMOs. Physical Chemistry Chemical Physics, 15(17):61666169, 2013.

[34] Miguel Jorge. Structure of cationic surfactant micelles from molecular simulations of self-assembly. Journal of Molecular Structure: THEOCHEM, 946(1):88-93, 2010.

[35] HJC Berendsen, JR Grigera, and TP Straatsma. The missing term in effective pair potentials. Journal of Physical Chemistry, 91(24):6269-6271, 1987.

[36] A Ramstad, G Brocks, and PJ Kelly. Theoretical study of the Si (100) surface reconstruction. Physical Review B, 51(20):14504, 1995.

[37] Paolo Giannozzi, Stefano Baroni, Nicola Bonini, Matteo Calandra, Roberto Car, Carlo Cavazzoni, Davide Ceresoli, Guido L Chiarotti, Matteo Cococcioni, Ismaila Dabo, et al. QUANTUM ESPRESSO: a modular and open-source software project for quantum simulations of materials. Journal of Physics: Condensed Matter, 21(39):395502, 2009.

[38] Norman Troullier and José Luriaas Martins. Efficient pseudopotentials for plane-wave calculations. Physical Review B, 43(3):1993, 1991.

[39] Stefan Grimme, Jens Antony, Stephan Ehrlich, and Helge Krieg. A consistent and accurate ab initio parametrization of density functional dispersion correction (DFT-D) for the 94 elements H-Pu. The Journal of chemical physics, 132:154104, 2010.

[40] Ilian T Todorov, William Smith, Kostya Trachenko, and Martin T Dove. DL_POLY_3: new dimensions in molecular dynamics simulations via massive parallelism. Journal of Materials Chemistry, 16(20):1911-1918, 2006. 
[41] Fredrik Tiberg, Johanna Brinck, and Lachlan Grant. Adsorption and surface-induced self-assembly of surfactants at the solid-aqueous interface. Current opinion in colloid $\&$ interface science, 4(6):411-419, 1999.

[42] Hirokatsu Miyata and Kazuyuki Kuroda. Preferred alignment of mesochannels in a mesoporous silica film grown on a silicon (110) surface. Journal of the American Chemical Society, 121(33):7618-7624, 1999. 